\title{
A comparison of brazed metal and epoxied fibre Bragg grating strain sensors under high strain regimes
}

\author{
I. Mckeeman*a, P. Niewczas ${ }^{\mathrm{a}}$, S. Khan ${ }^{\mathrm{b}}$ \\ ${ }^{a}$ Department of Electronic and Electrical Engineering, University of Strathclyde, Glasgow UK \\ ${ }^{\mathrm{b}}$ Civil Design Group, EDF Energy, Nuclear Generation, East Kilbride, United Kingdom \\ *iain.mckeeman@strath.ac.uk
}

\begin{abstract}
Three different fibre Bragg grating strain sensors were tested for their suitability for measuring high strain. The sensor types were: a bare epoxied fibre Bragg grating, a standard fibre Bragg grating epoxied into a metal capillary and, finally, a metal coated fibre Bragg grating brazed into a metal capillary. The sensors were calibrated on a steel specimen up to $1400 \mathrm{MPa}$ (equivalent to $7.3 \mathrm{~m} \varepsilon$ ). The results show that the bare epoxied Bragg grating and the metal packaged grating are suitable for measuring strains of this level.
\end{abstract}

Keywords: prestress monitoring, fibre Bragg gratings, structural health monitoring.

\section{INTRODUCTION}

Structural health monitoring is increasingly employed on large structures that are either safety critical or valuable assets such as nuclear containment buildings and long-span bridges [1]. There are numerous advantages of recording the real time response of the strain, temperature and acceleration of these structures. Optical sensors are capable of measuring such quantities with well-known advantages like multiplexibility and immunity to EM interference [2]. Fibre Bragg gratings (FBGs) are one of the most common methods for optically measuring strain and temperature. FBGs are written directly into optical fibre and consequently must be packaged in a proper manner if they are to survive deployment in a practical scenario. Different applications require appropriate packaging methods, for example when monitoring high strain in nuclear applications it might be beneficial for the FBG to be packaged without using epoxy [3].

This paper compares three different strain sensors for their suitability in practical high strain structural health monitoring applications. Two of the sensors consisted of an FBG sealed inside a metal capillary. One had an all metal construction; a metal coated fibre was brazed into the metal capillary with high-temperature metal solder, while the other was a standard optical fibre epoxied into the capillary. We show that the packaged FBGs can survive stresses of up to $1400 \mathrm{MPa}$ (equivalent to $7.3 \mathrm{~m} \varepsilon$ ). While the sensitivity of the packaged FBGs was lower than a bare epoxied FBG (the third sensor), they are more suitable for practical applications due to the ease of attaching them to the structure. Furthermore the metal brazed sensor wavelength response was more repeatable than that of the epoxy-capillary sensor.

\section{THEORY}

The peak wavelength of an FBG reflection spectrum can be measured to infer temperature or strain. In the case of an FBG that is attached to a steel specimen, the change in wavelength $(\Delta \lambda)$ of the FBG when subjected to mechanical strain $\left(\varepsilon_{m}\right)$ without temperature variations, as was the case in this experiment, is given by the equation [4]:

$$
\frac{\Delta \lambda}{\lambda_{0}}=K_{\varepsilon}\left(1-\rho_{e}\right) \varepsilon_{m}
$$

Where $K_{\varepsilon}$ and $\rho_{e}$ are the strain transfer between the specimen and the FBG and the photo-elastic coefficient respectively.

\section{SENSOR FABRICATION}

\section{Epoxied FBG}

A standard FBG written in SMF-28 fibre, with no recoating, was bonded directly to the specimen (Figure 1b) with EPOTEK 301 epoxy and cured at $65^{\circ} \mathrm{C}$ for two hours. The epoxy was applied along the length of the FBG. This method of 
attachment removes the influence of sensor packaging on the strain sensitivity of the FBG, leaving only the strain transfer between the epoxy and the specimen. However this could lead to inconsistencies between multiple sensors due the epoxy curing and the amount applied. The epoxied FBG must also be protected as the interrogating fibre will be exposed. The epoxied FBG enabled the effect of the sensor package on the strain transfer to the FBG to be investigated.

\section{FBG epoxied into sensor package}

A second type of FBG strain sensor was attached to the steel specimen (Figure 1a). A standard fibre FBG was epoxied (with EPO-TEK 301 epoxy) into a kovar capillary. Epoxy was applied to the length of the FBG and at both ends of the capillary; the FBG was then inserted into the capillary and pulled back and forth in the capillary to ensure the epoxy coated the FBG evenly. Steel shim was brazed onto the capillary prior to the FBG being epoxied into it. The sensor was then spot welded onto the steel specimen. This type of sensor provided a direct comparison for the metal packaged sensors that have been developed previously [3]. The shims were slightly closer together on the capillary compared to the metal sensor so that brazing paste did not cover the capillary holes where the fibre was inserted.

\section{FBG brazed into sensor package}

The third sensor attached to the steel specimen had an identical sensor package but the fibre inserted into the capillary was metal coated and the fibre was brazed into the capillary (Figure 1a)[3]. This sensor was also spot welded to the specimen.
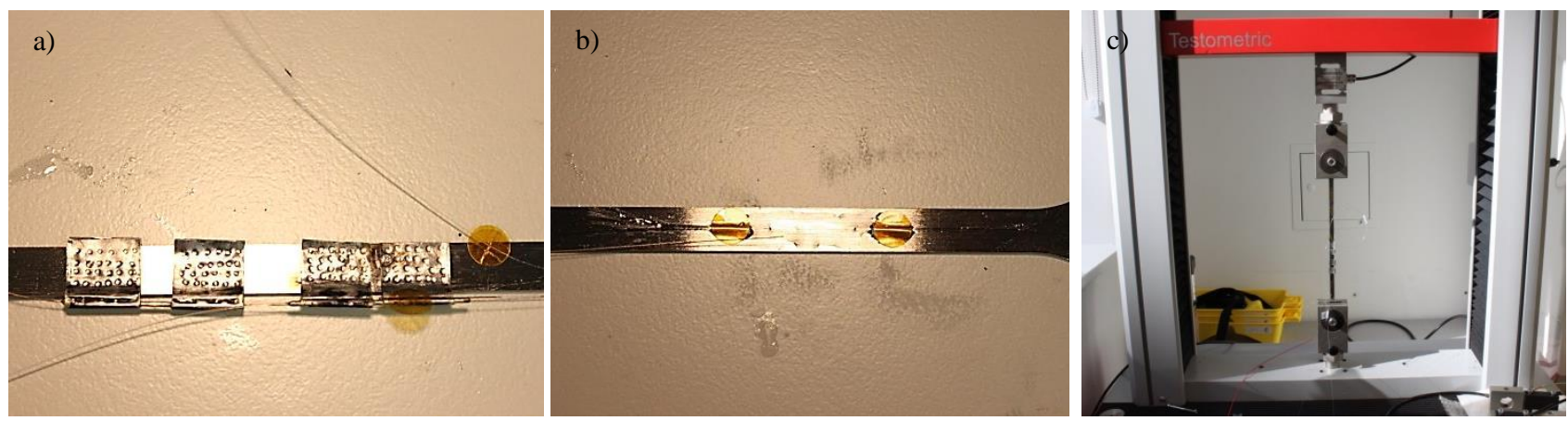

Figure 1. The attached packaged FBG strain sensors can be seen in 1a. Although they look similar, the sensor on the left of the photo had the FBG brazed into the capillary with metal brazing paste while the one on the right had the FBG epoxied into the capillary. The final sensor (1c) was a bare FBG epoxied onto the steel specimen. Photo 1c shows the steel specimen, with sensors attached, mounted on the tensile machine.

\section{SENSOR TEST METHOD}

The sensors were attached to a high strength (CS95 grade) steel dumbbell specimen. The steel had a Rockwell hardness of 50-52, equivalent to a tensile strength of $1663-1725 \mathrm{MPa}$. The dimensions were chosen so that the steel could be stressed upwards of $1400 \mathrm{MPa}$ on a $10 \mathrm{kN}$ tensile machine; this resulted in the steel having a width and thickness of $7 \mathrm{~mm}$ and $1 \mathrm{~mm}$ respectively. The required stress was achieved by tensioning the specimen to a force of $9.8 \mathrm{kN}$.

The steel specimen was mounted on a $10 \mathrm{kN}$ tensile machine (Figure 1c). A test profile was programmed for the machine to increase force in multiples of $1 \mathrm{kN}$ (up to $10 \mathrm{kN}$ ), hold the force for 20 seconds and then return to $0 \mathrm{~N}$ in $1 \mathrm{kN}$ steps, see Figure 2a. This was repeated for three cycles. The FBG sensors were interrogated continuously during the test on separate channels of a National Instruments PXI-4844 interrogator. Force measured by an external load cell was also recorded. Additionally, temperature was recorded by a thermocouple to compensate the strain sensors. The temperature during the experiment changed by $0.02^{\circ} \mathrm{C}$, the strain sensors had an average temperature sensitivity of $15 \mathrm{pm} /{ }^{\circ} \mathrm{C}$. The National Instruments interrogator has an accuracy of $1 \mathrm{pm}$ therefore the result of this temperature change was negligible, especially considering the wavelength changes due to stressing the sensors.

In order to calibrate the strain sensors, the load cell force was converted to strain using the Young's modulus of the specimen $(193 \mathrm{MPa})$. Figure $2 \mathrm{~b}$ shows the stress-strain relationship of the specimen, showing that the stress to strain conversion was relatively linear across the force range; therefore this is an appropriate approximation. Figure $2 b$ also shows that the extension measured by the machine was possibly unreliable because a larger than expected strain was recorded. This was because its reading includes extension of the machine grips. The load cell measurement has a better 
accuracy and this is why it was used for the calibration. Future work would benefit from using an external extensometer. The converted strain was compared with the wavelength measurements from the three sensors. The wavelength and strain readings used for the calibration were taken midway through the 20 second period when the tensile machine was holding a given force. A line of best fit using the least squares method was applied and from this the strain transfer $\left(K_{\varepsilon}\right)$ of the individual sensors could be calculated. This assumed a perfect strain transfer would change the wavelength of the FBG by $0.78 \varepsilon$ (see Theory section).
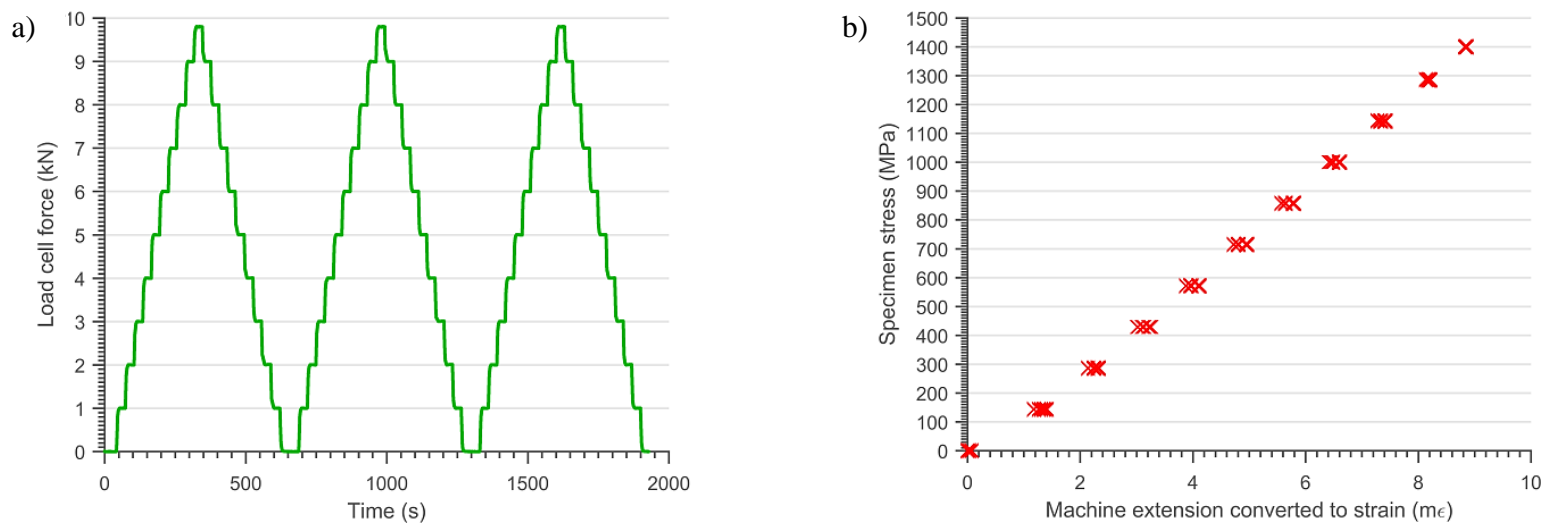

Figure 2. The test profile for the experiment, 2a. Stress against strain measured by the machine, $2 \mathrm{~b}$. Note that the extension measurement (converted to strain) is possibly unreliable; future work will benefit from an external extensometer.

\section{RESULTS AND DISCUSSION}

Figure 3 shows the results of the calibration procedure for the three sensors. The epoxied FBG wavelength response can be seen in 3a. The response was linear up to $1400 \mathrm{MPa}$ which is equivalent to $7.3 \mathrm{~m} \varepsilon$. The least squares fit for the three cycles shows that the sensor reading was very repeatable. The sensitivity is high, with a strain transfer of about $95 \%$. This is beneficial for accurately measuring small changes in strain however there is a trade-off between sensitivity and the number of sensors that can be multiplexed. Additionally, from a practical perspective curing epoxy in an industrial environment, often in wet ambient conditions will likely impact the consistency of the attachment. Furthermore, without protection the epoxied sensor is vulnerable to damage as the fibre is exposed.

The FBG epoxied into a metal capillary did not perform consistently over the three cycles. For each cycle, the zero strain wavelength and the slope of the least squares fit changed, showing that the epoxy was not able to secure the optical fibre into the capillary to survive the applied stress. The strain transfer was about $1 \%$, therefore with a $1 \mathrm{pm}$ accuracy interrogator the minimum strain change that could be detected would be approximately $70 \mu \varepsilon$. Although this particular sensor cannot be reliably calibrated, additional sensors of this type need to be tested to ensure that this sensor was not faulty and that other sensors perform adequately.

Finally the calibration of the FBG brazed into a metal capillary can be seen in Figure 3c. The wavelength response of the sensor shows that it was repeatable over the $1400 \mathrm{MPa}$ range. There was some hysteresis that was repeatable and this could be included in the calibration. The wavelength change across the force range was about $1 \mathrm{~nm}$ leading to a strain transfer of $10 \%$. Although this was lower than the epoxied FBG, this also means more sensors can be multiplexed within a given bandwidth. Additionally the sensitivity is adequate for a strain resolution of less than $10 \mu \varepsilon$, which is acceptable for most applications. This sensor can be attached to a structure relatively quickly by spot welding.

From these results it is clear that in terms of strain sensitivity and repeatability the epoxied FBG performed the best, although the metal package sensor also had a repeatable wavelength response. However if the sensors are to be deployed in a practical scenario there are several considerations for deciding the most suitable sensor as mentioned above. Where high precision strain measurements are required, based on these results it would be advisable to epoxy a bare FBG directly onto the specimen, assuming this can be done. Whereas if a fast installation and additional fibre protection is needed the metal packaged sensor would be more suitable. 

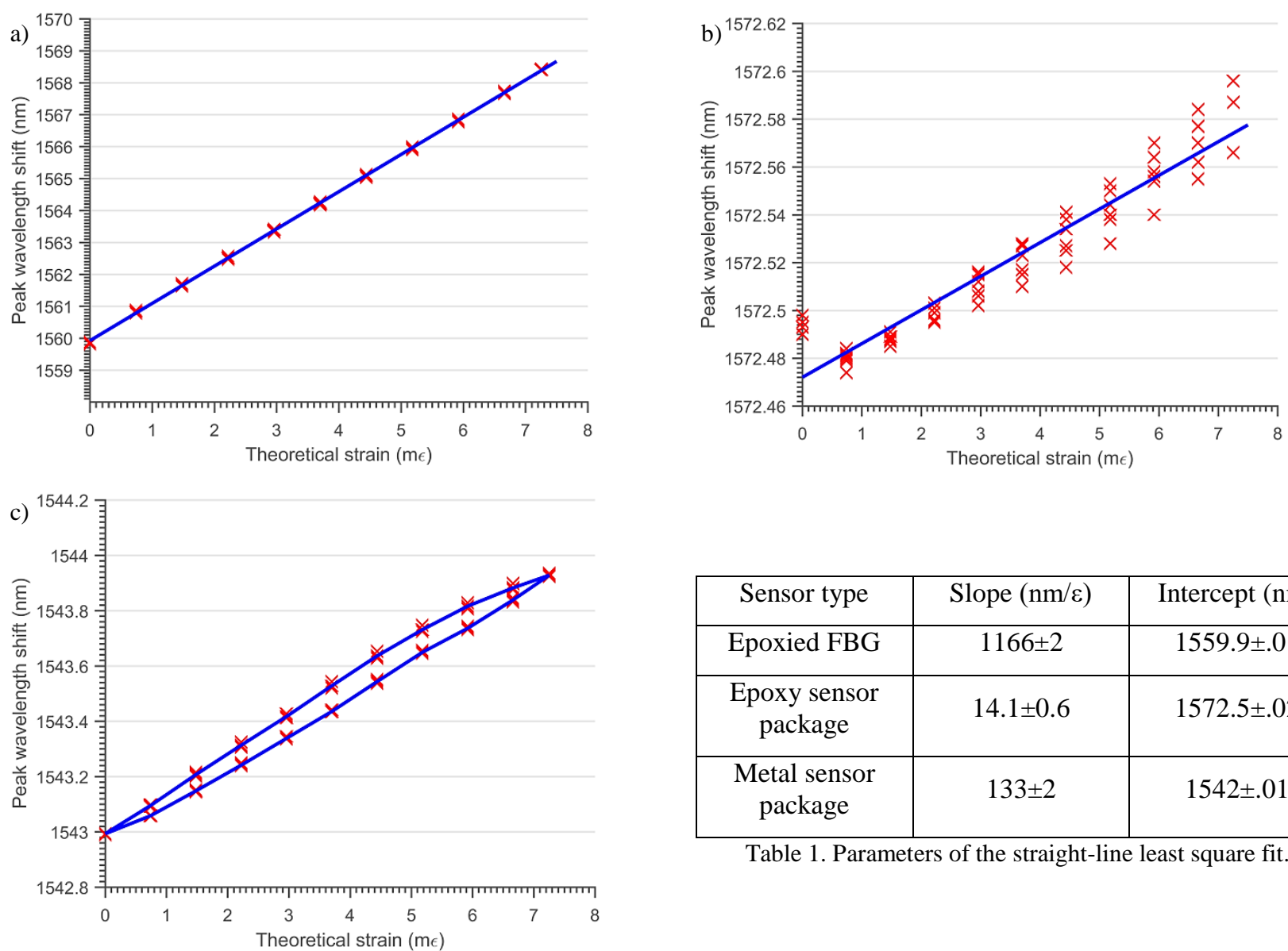

\begin{tabular}{|c|c|c|}
\hline Sensor type & Slope $(\mathrm{nm} / \varepsilon)$ & Intercept $(\mathrm{nm})$ \\
\hline Epoxied FBG & $1166 \pm 2$ & $1559.9 \pm .01$ \\
\hline $\begin{array}{c}\text { Epoxy sensor } \\
\text { package }\end{array}$ & $14.1 \pm 0.6$ & $1572.5 \pm .02$ \\
\hline $\begin{array}{c}\text { Metal sensor } \\
\text { package }\end{array}$ & $133 \pm 2$ & $1542 \pm .01$ \\
\hline
\end{tabular}

Table 1. Parameters of the straight-line least square fit.

Figure 3. Calibration of the epoxied FBG (3a), the epoxy sensor (3b) and the metal sensor (3c)

\section{CONCLUSION}

This paper compares three different types of FBG strain sensor. The sensors were calibrated up to 1400MPa, equivalent to $7.3 \mathrm{~m} \varepsilon$. Despite the high stress and strain, the epoxied FBG and metal packaged sensor wavelength responses were repeatable and these sensors have been shown to be suitable for measuring these high strains. We have also discussed some of the practical considerations for choosing the most suitable type of sensor. Further investigation is needed to determine if the FBG epoxied into the metal capillary was defective or if this technique is not suitable for fabricating strain sensors to monitor high strain.

\section{ACKNOWLEDGEMENTS}

This work was supported by the Engineering and Physical Sciences Research Council (EPSRC) and EDF Energy Nuclear Generation, Ltd.

\section{REFERENCES}

[1] J. M. Lopez-Higuera, L. Rodriguez Cobo, A. Quintela Incera, and A. Cobo, "Fiber Optic Sensors in Structural Health Monitoring," J. Light. Technol., vol. 29, no. 4, pp. 587-608, Feb. 2011.

[2] L. S. Grattan and B. T. Meggitt, Optical Fiber Sensor Technology: Fundamentals. Springer Science \& Business Media, 2013.

[3] I. Mckeeman et al., "First-time demonstration of measuring concrete prestress levels with metal packaged fibre optic sensors," Smart Mater. Struct., vol. 25, no. 9, p. 95051, Sep. 2016.

[4] M. Kreuzer, "Strain measurement with fiber Bragg grating sensors," HBM, Darmstadt, Germany. 